\title{
DISTRIBUTION FUNCTIONS OF TYPE III ELECTRONS OBSERVED IN INTERPLANETARY SPACE
}

\author{
R.P. Lin, D.W. Potter, K.A. Anderson \\ Space Sciences Laboratory, University of California, \\ Berkeley, California 94720 \\ J. Fainberg, R.G. Stone \\ Goddard Space Flight Center, Greenbelt, Maryland 20771 \\ J.L. Steinberg \\ Department de Recherches Spatiales, Observatoire Meudon, \\ 92190 Meudon, France
}

We present simultaneous energetic electron and solar radio observations from the ISEE-3 spacecraft of several solar type III radio bursts. The UC Berkeley energetic particle experiment measures from 2 to $\sim 10^{3} \mathrm{keV}$ with good energy and pitch angle resolution while the Meudon/GSFC radio experiment tracks type III radio bursts at $24 \mathrm{fre}-$ quencies in the range $30 \mathrm{kHz}-2 \mathrm{MHz}$.

Two types of electron events are observed by the particle experiment: (1) the "normal" type of 20 to > $100 \mathrm{keV}$ flare associated electron events reported previously, and (2) events where only electrons in the 2-10 keV range are observed. These low energy, 2-10 keV, electron events appear not to be associated with flares or high frequency ( $>10 \mathrm{MHz}$ ) radio emission as reported in the Solar Geophysical Data bulletins, but rather they appear to be associated with individual type III bursts from low frequency type III storms. Both types of electron event are closely correlated to the very low frequency type III emission produced in situ at $1 \mathrm{~A} . \mathrm{U}$. The radio experiment is able to identify the direction of arrival of the radio emission through a combination of a dipole antenna spinning in the ecliptic plane plus z-axis antennas for out-of-the-ecliptic direction. The frequency of the radio emission produced in situ around the spacecraft can be identified by its omnidirectional nature. We have compared the temporal behavior of the in situ radio intensity and the electron intensity. In every case good agreement was found between the onset times for the in situ radio and electron events. For the single event where solar wind plasma density measurements were available the frequency of the radio emission is consistent with the emission being generated at the second harmonic and not at the fundamental of the plasma frequency.

We have made preliminary computations of the electron velocity distribution function parallel to the magnetic field, $f\left(v_{\|}\right)$, for seyeral events. In the $>20 \mathrm{keV}$ events no strong positive slope $d v_{11}$ II) $>0$ is observed, rather $f\left(v_{\|}\right)$has a plateau-like shape, even 
though a peak is observed in the energy spectra. The plateau appears to be due to the fact that the pitch angle distribution is broad, not beamlike. However in the low energy 2-10 keV electron events a peak is observed in the parallel velocity distribution function with $\frac{\mathrm{df}\left(\mathrm{v}_{\|}\right)}{\mathrm{dv_{ii }}}>0$ since the pitch angle distribution is very narrow, FWHM $<22.5^{\circ}$, and beamlike. It appears that above $20 \mathrm{keV}$ scattering in pitch angle may play an important role in modifying the parallel velocity distribution function.

\section{DISCUSSION}

Papadopoulos: Your strong pitch angle distributions can be atrribut-ed to the fact that only the front of the beam is resonantly unstable. The back of the beam is nonlinearly stable. However in propagating from the Sun to 1 AU it sees the strong turbulence clumps, like heavy ion clumps, from which it can pitch angle scatter very effectively and appear as a plateau at $1 \mathrm{AU}$, with no energy loss.

Vlahos: Since you mention that these events are related to type III storms can you exclude the possibility that the plateau is an accumulation effect?

Lin: The low energy $\leq 10 \mathrm{keV}$ events which do not have a plateau are the events which are related to type III storms. The $>20 \mathrm{keV}$ events, which have plateau-like distributions, are not related to type III storms.

R.A. Smith: There may be a statistical difficulty in observing the unstable portion of the beam, as the distribution should be highly structured in space, owing to the interaction with solitons.

Hudson: Do you have a simple explanation for the absence of long decay times at low energies? If they are less diffusive, perhaps they can be used to probe conditions at the acceleration site more directly.

Lin: I have a naive experimentalist's explanation for the lack of scattering at low energies. It may be due to lack of waves of the scale size resonant with those particles. Thermal protons in the solar wind have gyro-radii comparable to that of $\sim 20 \mathrm{keV}$ electrons. Thus one might expect waves resonant with $\gtrsim 20 \mathrm{keV}$ electrons to be present. However, at smaller scale sizes comparable to < $10 \mathrm{keV}$ electron gyro-radii there is perhaps no comparable source of waves.

Bradford: What is the background electron spectrum that the events are superimposed on?

Lin: The background electron spectrum is the quiet time spectrum which is always observed in the interplanetary medium. Its origin is not known but at least at low energies ( $<10 \mathrm{keV}$ ) appears to corne from the solar direction. 
Bradford: Then it is not a long lived electron event.

Lin: No

Takakura: Have you checked the growth rate $\gamma(v)$ of the plasma waves by using your results of $\partial \mathrm{f}(\mathrm{v}) / \partial \mathrm{v}$ ? $\Gamma=\int \gamma(\mathrm{v}) \mathrm{dt}$ of the order of 20 is required to have strong enough plasma waves. It means that a rather remarkable hump in $f(v)$ may remain near 1 AU.

our $\frac{\mathrm{din}}{\frac{\mathrm{d}}{\mathrm{dv}}}$ measurements.

Benz: Electron beams can couple to electromagnetic ion-cyclotron waves. This resonance interaction has been investigated by Pilipp and Benz (Astron. Astrophys.) in relation to type $V$ metric radio bursts. It seems possible that the scattering in pitch angle you observed may be due to this interaction. I suggest that you look for ion-cyclotron waves in ISEE wave detector data.

Lin: We plan to look in detail at the plasma wave data, but I don't recall seeing ion cyclotron waves. 